The appendix was found curled on itself and adherent to the uterus. The left Fallopian tube was found to be inflamed and reddened. It was taken up with a purse-string suture of pyoktannin catgut, first being freed from intestinal adhesions. 'The tube and ovary were excised; the broad ligament being tied ahead of them, with a running suture of pyoktannin catgut. The appendix was freed from its mesentery and the serous membrane incised circularly with a knife about onequarter of an inch above the attachment to the intestine; it was ligated close to this attachment and cut off where the circular incision was made. The stump was cauterized with carbolic acid and alcohol; a purse-string suture of pyoktannin catgut taken round it and the stump inverted and suture drawn. The patient was discharged, well, Sept. 30, 1902.

CaSE 15.-A. W., aged 28, single, was admitted to hospital July, 1903.

History.-In the past three years she has been troubled with pains in her pelvis. She began to menstruate and has had discharge ever since. She does not flow much during the menstrual period. About a week previously she began to have chills and sharp pains in the lower part of abdomen. She was in bed two days; then got up and pain began in back, whicb at last localized to one spot with a burning sensation. This was on the right side. Bowel movements caused pain.

Diagnosis.-Pyosalpinx and ovaritis of right side.

Operation.-Laparotomy: Salpingo-oöphorectomy. An incision was made in the median line, about four inches long, between the umbilicus and the pubes. Intestines, right tube and uterus were matted together in Douglas cul-de-sac, and the appendix was adherent to the tube. Adhesions were gently broken up. The tube was twisted and kinked, being very thick and irregular nearest the uterus; a pyoktannin, No. 2, ligature was passed round it, near the uterus, and tied and the uterine attachment cut. The ovary was grasped with a pair of forceps, but its pedicle was torn and the ovary was removed and torn. The pedicle was sutured with No. 2 pyoktannin catgut and the suture continued along the broad liga. ment at the uterine attachment, which was cut loose. The suture was continued by a needle passed through a loop of the preceding stitch. The mesoappendix was cut and then sutured in the same way as the broad ligament, and the peritoneum of the appendix was incised all around at the base and folded down as a cutf; the appendix was ligated about onequarter of an inch below with the same kind of catgut and then cut a short distance from ligature, the end being touched first with 95 per cent. carbolic acid and then with alcohol The open end was closed with No. 0 pyoktannin catgut and the peritoneal cuff folded over the end and sutured with the same. The peritoneum was closed with continuous suture, No. 2, and fascia and muscles in the same way. The skin was sutured with subcuticular silkworm gut. After closing it the line of suture was irrigated with hot normal salt solution. The patient was discharged well Aug. 22, 1903.

CASE 16.-A. F., aged 22, married, was admitted to the hos. pital June 24, 1903.

History.-The patient has had one child, which died when 4 days old. About two weeks ago she had severe pains in the lower abdomen on both sides. About five days ago pains on left side stopped, but pain on right side persists. She says that when she urinates she has bearing-down pain on right side.

Diagnosis.-Double pyosalpinx (gonorrhea).

operation.-Laparotomy: Salpingo-oöphorectomy. The abdomen was opened in the median line. On opening the peritoneal cavity, a large mass, consisting of adherent omentum, was brought into view. On investigation of the right ovarian region, another mass about the size of the first was discovered bound down with firm adhesions and lying more in the abdominal than in the pelvic cavity. This mass was separated from surrounding intestines, which later were walled off with gauze pads. Omental and intestinal adhesions were tied and cut. The pedicle of the mass was transfixed with needle, tied off with pyoktannin catgut and cut. The mass was lifted up, but ruptured before it could be removed. It proved to be a tubo-ovarian abscess, containing almost a cupful of yellowish pus. The pus was sponged out; the stump and edges of the broad ligament were brought together and sutured, a running suture of catgut being introduced. These stitches tore out and later had to be reintroduced. The left side was investigated and another tubo-ovarian abscess was found and treated in the same way, except that a portion of the ovary was dissected from the mass and allowed to remain. The pelvic and abdominal cavities were sponged dry; the peritoneum was closed with catgut. Muscle, fascia and aponeurosis were closed with Billroth's chain suture, pyoktannin catgut being used. The skin was closed with subcutaneous stitch of medium silkworm gut. Patient was discharged, well, Aug. 31, 1903.

( $T o$ be continued.)

\section{DIAGNOSIS OF AFFECTIONS CHARACTERIZED BY INTESTINAL OBSTRUCTION.}

\author{
G. PAUL LA ROQUE, M.D. \\ Lecturer and Bedside Instructor in Surgery at the University \\ College of Medicine. \\ RICHMOND, VA.
}

Since the clinical phenomena presented by incomplete or partially obstructive lesions in this tube are referable, rather to the nature and location of the lesion than to obstruction, these affections will not be studied in this paper.

Complete obstruction to the passage of the contents of the intestinal canal is generally characterized clinically by the symptom-complex of an acute affection. The causative lesion, however, may be of acute (sudden) or chronic (slow) development. In accordance with the underlying clinically recognizable etiologic factor, the different varieties of acute intestinal obstruction (ileus) may be classified as (1) dynamic ileus and (2) mechanical obstruction.

1. Dynamic Ileus.-This is due to peristaltic paralysis either of the entire intestine or of a segment of gut and is typified by the absolute intestinal obstruction incident to general peritonitis. It is not rarely due to mechanical and chemical irritation of the peritoneum, such as is incident to extensive operations about the mesentery, vigorous toilet of the peritoneum, prolonged rough handling, chilling and exposure of this structure and was common in the days of antiseptic abdominal surgery under the use of carbolic acid spray and other antiseptics in the peritoneal cavity. Contusion of the peritoneum due to external violence is a cause. It may also result from excessive meteorism due to enteritis. Intestinal paresis may result from splanchnic and spinal nerve degeneration, cord injury, and is a common terminal condition often directly causative of death in certain systemic diseases, particularly pneumonia, uremia, meningitis, typhoid fever and others, and especially when associated with delirium, unconsciousness, coma and excessive pain and other symptoms which at the same time divert the attention from the abdomen and predispose to constipation and to meteorism. In mild form transient paretic ileus is noted after the removal of large abdominal tumors and ascites, and is the rule after childbirth. The temporary reflex intestinal paresis incident to injury of a testicle, operative procedures about the anus and rectum, during a paroxysm of severe renal, ovarian, biliary or pancreatic colic, and often conspicuous in the symptomatology of these affections, is non-progressive is severity and transient. In many cases of mechanical obstruction, particularly when due to strangulation, there may be initial reflex paresis of the entire gut, though the proximal portion soon recovers. and becomes hyperactive against the strangulated para- 
lyzed segment which constitutes a mechanical obstacle to peristalsis.

2. Mechanical Obstruction.-This may result from strangulation of a segment of intestine or its mesentery through occlusion of its blood vessels, the strangulated portion of gut constituting an obstacle to or solution of continuity of the peristaltic wave. It is typified by the ileus incident to mesenteric embolism and thrombosis, torsion of the mesentery, constriction of the blood vessels by bands, adhesions, hernial orifices and traction, and the early stages of incompletely occlusive invagination (intussusception). Mechanical occlusion, either by obturation or stenosis of the lumen of the intestine, is the typical cause of mechanical obstruction. Obturation results from impaction of gallstones, entoroliths, fecal material and innumerable varieties of foreign material introduced either by way of the mouth or per anum. Stenosis may result from stricture formation, cicatrical or neoplasmic in character, constriction from without by bands of peritoneal adhesions, any abdominal or pelvic tumor, distended and displaced organs and finally rolvulus (torsion) or angulation (kinking).

\section{r. DYNAMIC ILEUS.}

Cardinal symptoms: (1) In all cases the signs of pseudoperitonitis or peritonitis are present and coincide with or antedate those of obstruction. (2) Absolute constipation to both feces and flatus exists, and with this (3) absent peristalsis. (4) Pernicious vomiting, becoming stercoraceous in character, supervenes. (5) Progressive uniformly distributed abdominal tympany becomes extreme. (6) Pain, at first, is that of the causative factor. As distention increases, general abdominal discomfort, costal breathing and shortness of breath are noted. These are ameliorated by belching of gas and evacuation through the rectal tube. True intestinal colic does not occur with absent peristalsis, though this symptom may precede those of obstruction. Other forms of colic must not be mistaken. When peritonitis is the cause (and this is the typical and most important variety of dynamic ileus) the pain of this affection antedates the signs of obstruction and continues till toward death. (7) Constitutional depression is generally conspicuous. Shock may exist as a coincident symptom or may be absent. (8) The urine is scanty, concentrated and contains an excess of indican if the condition is as much as twenty-four hours in duration.

\section{MECHANICAL OBSTRUCTION.}

The signs common to mechanical ileus may conveniently be enumerated in two groups, (A) strangulation; (B) obturation.

A. Strangulation Ileus: The signs of this condition are divisable into those of $(a)$ strangulation per se, $(b)$ peristaltic obstruction and intestinal distension, $(c)$ gangrene of gut, $(d)$ peritonitis.

(a) Strangulation per se.-(1) Sudden, violent, agonizing general abdominal pain is due to nerve irritation; (2) persistent nausea and vomiting are reflex in origin; (3) profound shock is noted; (4) peristalsis is commonly reflexly diminished in intensity or paralyzed. To these is soon added: (5) urgent desire to defecate, of ten to the degree of tenesmus. One or two good bowel movements may or may not occur, mucus and often blood are later ejected.

(b) peristaltic Obstruction and Intestinal Distention. - (6) Constipation becomes absolute to both feces and flatus; (7) nausea and vomiting increase in severity, becoming finally stercoraceous. (8) Intestinal tympany is first noted in the segment of gut strangulated, later in all the intestine above this seat. Distention of the distal portion of the bowel does not occur. (9) Exaggerated peristalsis is often visible, always audible as tumultuous sounds. (10) Pain is distinctly colicky in character and presents remissions and exacerbations in intensity.

(c) Gangrene.-With this there is: (11) shock or collapse; (12) diminution, often subsidence of pain; (13) enfeebled but still present peristalsis; (14) foul breath, coated tongue, headache, rise of temperature to normal or slightly above. These are all due to absorption of toxines (stercoremia).

If unrelieved these symptoms are soon followed by:

(d) Peritonitis-This is caused by intestinal permeability, perforation or rupture, and is heralded by (16) sharp pain (may be absent); (17) reflex rigidity; (18) fever; (19) leucocytosis; (20) all the signs of dynamic ileus (see above); (21) soon the evidence of free fluid in the peritoneal cavity; (22) peritoneal facies.

B. Obturation Ileus: The signs of this are dirisible into local obstruction to the lumen of the gut, proximal distention, stercoremia (autointoxication), intestinal rupture. In this condition we note: (1) Constipation. This is sudden and generally absolute, though the lower bowel may be emptied. Fàlse diarrhea may occur. (2) Intestinal colic. (3) Exaggerated peristalsis is risible and audible. (4) Proximal progressive tympany; and (5) nausea and vomiting are all pronounced, progressive and finally vomiting becomes stercoraceous. (6) Foul breath, coated tongue and moderate rise of temperature. (7) Finally, the signs of intestinal rupture are engrafted and the clinical picture radically altered. Distention becomes uniform and peristalsis paralyzed. The patient, if conscious, may have felt a sensation of rupture. There is frequently a history of the administration of cathartics.

Given a case of mechanical intestinal obstruction, the following table will be useful in differentiating stranguJation from occlusion:

TABLE FOR DIFFERENTIATING STRANGULATION FROM

$$
\begin{aligned}
& \text { OCCLUSION. } \\
& \text { STRANGULATION. OCCLUSion. }
\end{aligned}
$$

Generally the first sy m p tom. A later symptom. Less sudden, Sudden, violent, continuous, less violent at first but prolittle chance to progress in gressive and paroxysmal. Due severity, may subside with on- to intestiual spasm (colic).

set of gangrene. Due to nerve irritation.

$$
\text { D.--vomisixg and NaUsea. }
$$

Initial symptoms, reflex in char- Later symptom, due to overfillacter, less definitely stercora- ing of the canal. Stercoraceous ceous. Death may occur before material is sure to be noted feces are vomited. in late stages. c. - SHOCK.

Sudden, pronounced and rapidly May not be noted early and is progressive.
of gradual development. Due sieeplessness, starvation and exhaustion.

D.-BOWEL ACTION.

Peristalsis in earliest stages is $\mathrm{Per}$ is t a $1 \mathrm{sis}$ progressively inreflexly diminished. False diar- creased from the beginning rhea with mucus and blood in False diarrhea is less rare and passages may be noted in may be conspicuous. No blood mesenteric embolism and in in passages though watery intussusception. mucoid material may be
ejected.

E.-INTESTINAL TYMPANY.

Noted first in the Ioop of gut No circumscribed distention of strangulated and then becomes loop, distention is general general. from the first.

F.-AUTOINTONICATION.

The signs of this are obscured These signs are conspicuous long by those of gangrene and peri- before peritonitis develops. tonitis. G. CLINICAL COURSE.

Always violently acute and rap- May be acute, often chronic, idiy followed by gangrene and with recurrent exacerbations peritonitis unless relieved. dependent on the cause (see below). Peritonitis is late gangrene may not occur. 
Diagnosis of Cause of Mechanical Obstruction. A. Strangulation.

In all cases of sudden abdominal pain with violent vomiting and constipation, especially after some sudden muscular effort as heavy lifting, all the hernial orifices must be examined. In one known to have a hernia, strangulation is the most probable cause.

Strangulated Hernia.-The obstruction symptoms are those of strangulation ileus (see above). Pain may be felt at the point of strangulation, the hernia becomes painful, tender, enlarged, generally irreducible, dull to percussion, the impulse on coughing is lost. If only the omentum is strangulated, constipation is less absolute. Sudden cessation of pain points to gangrene or to reduction. Strangulation must be differentiated from incarcerated and from inflamed hernia. In neither of the latter is impulse on coughing lost. In incarcerated hernia the gastrointestinal signs are of a mild imperfect obturation of the bowel. In inflamed hernia the signs are of local inflammation, with perhaps localized peritonitis, but never positive ileus.

On excluding strangulated external hernia the signs referable to the internal hernial orifices should be sought. Diaphragmatic, obturator, sciatic, duodenojejunal and retroperitoneal hernia must be suspected.

Diaphragmatic Hernia.--In this there are, in addition to the signs of strangulation ileus with little or no abdominal distention, the signs of suddenly developing pneumothorax, generally on the left side, without pulmonary or pleural cause. Vomiting is never fecal. Obturator and sciatic hernias are characterized by pain in the region of these orifices, often referred down the thigh. Tenderness may occasionally be elicited on rectal examination. These, together with retroperitoneal, duodenojejunal and other forms of internal hernias, are generally unrecognizable save as strangulation ileus.

Strangulation and Occlusion by Bands or Adhesions. -In about 70 per cent. of patients there is a history of previous peritonitis (due to appendicitis, salpingitis or previous abdominal section) though this may have to be interpreted from previous attacks of colic, etc., rather than by knowledge of the patient. It is extremely rare in childhood. The signs are of strangulation and mechanical ileus, with complete or incomplete occlusion (see above). Constipation is absolute; there is, as a rule, no tenesmus, nor is bloody mucus passed from the empty rectum. Stercoraceous vomiting is late. Distention is gradual and central (involving chiefly the small gut). A fixed, distended paralyzed loop of intestine may be discovered. The colon is apt to be empty and collapsed, causing lateral flattening as contrasted with central distention.

Mesenteric Embolism.-This form of obstruction is characterized by the signs of strangulation ileus, with intestinal hemorrhage, soon free fiuid in the peritoneal cavity and occasionally a palpable blood tumor between the layers of the mesentery. There may have been no previous disease of the intestine and no signs of portal engorgement. There must be a source of embolism, i. e., cardiovascular disease, generally valvular in character, and signs of simultaneous embolism in other parts (lungs, spleen, joints, etc.) must be sought.

Intussusception.-In this affection the signs of strangulation ileus predominate, even though there be complete occlusion of the lumen of the canal. It is almost confined to children, appearing in the midst of perfect health, though dysentery and straining diarrhea may precede and predispose to the condition. Extreme me- teorism is exceptional. Flatness in the region of the colon may be noted and in this situation a soft tumor may often be palpated. In rare cases the tumor may be of sausage shape, but this must not be expected. Occasionally the invaginated bowel may be palpated in the rectum or sigmoid. This must not be mistaken for the indurated circular rings incident to hypertrophy of the circular layer of muscle in the wall of the rectum attending chronic dysentery. Tenesmus is generally conspicuous, vomiting is exceptionally absent.

Volvulus is almost confined to individuals past middle age, generally the subject of chronic constipation. The large intestine is the portion involved. The sigmoid (left flank) is commonly the seat of a rounded, tympanitic, quiet tumor, and proximal intestinal distention rapidly becomes extreme. The signs are those of mechanical obstruction and constipation is absolute. Diarrhea, even of the false variety, is exceptional and passage of blood is rare. Tenesmus is common. Vomiting is occasionally absent.

\section{B. INTERNAL OBTURATION}

The most common cause of this is fecal impaction. There are the signs of atony of the bowel. The patient is generally anemic and the victim of chronic gastric catarrh. Constipation for days before the onset of acute symptoms is the rule. The signs are of less rapid onset. Proximal distention and hyperperistalsis are marked. A doughy tumor may be palpated in the colon; rectal examination generally discovers a mass of hardened feces. There may be tenesmus and false diarrhea incident to irritative colitis (stercoraceous) or to channeling of the fecal mass. A high enema is generally curative.

Gall-stone Impaction.- This is rare. It occurs in individuals of gall-stone age and generally a history of cholelithiasis may be elicited. The signs are of mechanical occlusion. In rare instances a hard tumor may be felt in the situation of greatest pain. Should this tumor be made to alter its position in the gut or move downward spontaneously the diagnosis may be justified. A fluoroscopic examination may be useful, but not conclusive as a diagnostic measure. The signs may remit in intensity, and spontaneous passage of the stone in the large intestine is curative.

Impaction of Enteroliths.-This is causative of internal obturation. The diagnosis can be made only in rare cases, in which the mass can be palpated per rectum. $X$-ray examination may be positive or negative. Impaction of other foreign bodies can be recognized only when a positive history is obtainable or the material can be shown by $x$-ray examination.

Obstruction by Stricture Formation.-The diagnosis of this as a cause depends on diagnosis of the stricture. The signs are those of mechanical ileus. Obstruction occurs always in the large bowel and toward the anus. Syphilitic and tubercular stricture are found in the rectum and sigmoid. Cancer occurs at one of the curves. Constipation is rarely absolute. Mucus and blood in the fecal matter is frequent. Pain on defecation is present when the disease is located in the rectum or sigmoid.

Congenital Obstruction.-This is caused by imperforate anus, rectum or upper bowel, or by fistulæ between the gut and bladder, uterus or vagina. The symptoms of obstruction occur a few hours after birth. If the anus is imperforate the perineum bulges when the child cries. If the anus is formed a finger in the rectum notes bulging higher up in the pelvic cavity. If a fistula exists between the bowel, bladder or uterus, meconium may be discharged through these channels. 
STUDY OF INDIVIDUAL SYMP'TOMS.

While in many cases of intestinal obstruction the exact nature and location of the lesion can not be positively recognized before operation, the study of individual symptoms is helpful.

Pain.-This is due first to nerve irritation, later distention and congestion, finally peritonitis. It is generally first referred to the umbilicus, rapidly becoming general. It is colicky in character when obstruction is incomplete (due to spasm); constant and of short aching character when occlusion is complete (due to distention and congestion). Extreme tenderness and true rigidity, being due to peritonitis, are rare in early stages.

Collapse and Vomiting.-In acute cases these signs are due to nerve irritation, and when early severe they point generally to high obstruction, tight strangulation or pinching of the omentum. When occurring late, toxemia is the main cause and low obstruction is suspected. Frequently repeated, easy vomiting, with moderate uniform distention and oliguria, point to high obstruction. True fecal vomiting always means obstruction either in the lower ileum or colon or paralysis of the bowel from peritonitis. When occurring later, less urgent and slowly becoming. stercoraceous, while abdominal distention is great, low obstruction or peritonitis is suspected. When obstruction is in the transverse colon the right loin is more distended than the left.

Constipation.-When this is absolute, even to flatus, it means complete obstruction and points to volvulus, acute strangulation by bands of adhesions or at a hernial orifice. In other causes of acute obstruction constipation may be incomplete. Tenesmus, with the passage of mucus and blood, generally points to location at least below the splenic flexure in individuals in middle age or past; to intussusception in children.

Central Distention, with Flattening of the Loins.This sign suggests a lesion of the small gut. Great lateral distention points to the transverse colon or sigmoid as the seat of disease. The greatest tympany is always above the obstruction.

Visible Peristalsis.-Hypertrophy of the muscular wall of the gut is indicated by this sign and is rare in acute obstruction. In all forms of mechanical ileus, peristalsis is greatly exaggerated on auscultation until peritonitis develops.

Rectal Capacity.-When the obstruction is at or below the sigmoid rectal capacity is diminished to one or two pints; when above this point the rectum may hold as many quarts.

A history of biliary colic suggests gall-stones as the cause; chronic constipation suggests impacted feces; previous peritonitis causes constriction from bands; tuberculosis, syphilis and dysentery cause obstruction by cicatricial contraction of healed ulcers. Typhoid ulcers do not constrict the gut.

In children the cause is most frequently intussusception. In middle and old age volvulus, bands and malignant disease are the causes. A doughy tumor in either colon points to fecal impaction, and this in every case must be excluded. In all cases the hernial orifices and rectum must be examined. Abdominal tumors must be sought. Often laparotomy is necessary to diagnose the cause positively.

Diaphragmatic hernia may give no abdominal distention, but signs of dyspnea, etc., aid the diagnosis. They must not be mistaken for pneumothorax.
DIFFERENTIAL DIAGNOSIS.

Mechanical obstruction must be differentiated from all forms of acute dynamic ileus. Particularly that due to torsion of the spermatic cord, acute epididymitis, injury of the testicle, violent paroxysms of ureteral colic (Dietl's crises). In all these the ileus is transient, being due to pain and is non-progressive. Acute pancreatitis, perforation or rupture of hollow viscera, rupture of an ectopic gestation sac, splenic infarction must not be mistaken.

All forms of intestinal obstruction must be differentiated from acute appendicitis, tabetic crises, acute dilatation and volvulus of the stomach. The pain and often bloody diarrhea attending visceral manifestations of the acute erythematous skin affections (Osler's disease) must not be mistaken for intussusception nor for mesenteric embolism.

Acute dilatation of the stomach has been observed after injuries sufficiently severe to cause paralysis of gastric motility, and in mild degree is seen as a result of paralytic overdistention. The cardinal sign is enormous distention as determined by physical examination. There are epigastric pain and tympanitic tumor. Vomiting occurs from overflow, but when the distention is gaseous from motor nerve paralysis this sign may be absent. Regurgitation of gas occurs and is productive of relief. The affection in severe form is extremely rare.

A few cases of volvulus of the stomach are on record. It is characterized by acute agonizing abdominal pain. most severe in the epigastric region, accompanied by shock, persistent violent nausea, with retching and vomiting of small quantities of material from the stomach. A tense tympanitic tumor in the epigastrium may or may not be discovered. Vomiting is never stercoraceous and constipation is not in any way characteristic.

Acute pancreatitis is characterized by sudden, violent, agonizing, shocking pain, most severe in the upper abdomen, attended by severe reflex vomiting. Abdominal distention rapidly occurs, beginning in the epigastric region, soon becoming general. There is epigastric rigidity and tenderness, and often if these two signs can be overcome a deep-seated mass may be palpated. Constipation generally exists at least temporarily, but is not absolute, and can be overcome. Steatorrhea is a later sign, glycosuria may or may not occur. There is generally moderate fever in spite of shock, and if the latter should subside the temperature rises higher. Dyspnea and cyanosis are often observed. Slight jaundice from co-existing catarrh of the biliary passages may be present. Unrelieved there occurs rapid gangrene, peritonitis and death.

The subjects affected are generally males about middle life who have suffered with chronic dyspepsia or obstruction biliary disease. Slight traumatism may be the exciting cause. Abdominal section discovers fat necrosis in the mesentery if the condition is of twelve or twentyfour hours duration (?).

Acute pancreatitis must be differentiated from mechanical intestinal obstruction, biliary colic, suppurative cholecystitis, rupture of the gall bladder, stomach, intestine and other abdominal organs, acute appendicitis, torsion of an ovarian cyst or spermatic cord, injury of the testicle, tabetic crises and all abdominal affections characterized by acute agonizing pain attended by shock.

506 East Grace Street.

Medicine.-Osler says that medicine is the only world-wide profession following everywhere the same methods, actuated by the same ambitions, and pursuing the same ends. 